\title{
ABSTRACT \\ DISSOCIATION OF RIGHT AND LEFT HAND GESTURE SPACES IN SPLIT-BRAIN PATIENTS
}

\author{
Hedda Lausberg ${ }^{1,2}$ and Sotaro Kita ${ }^{2}$ \\ ( ${ }^{1}$ Department of Neurology, Free University of Berlin, Germany; ${ }^{2}$ Max Planck Institute for \\ Psycholinguistics, Nijmegen, The Netherlands)
}

\section{INTRODUCTION}

The present study investigates hemispheric specialisation in the use of space in communicative gestures. For this purpose, we investigate split-brain patients in whom spontaneous and distinct right hand gestures can only be controlled by the left hemisphere and vice versa, the left hand only by the right hemisphere. On this anatomical basis, we can infer hemispheric specialisation from the performances of the right and left hands. In contrast to left hand dyspraxia in tasks that require language processing, split-brain patients utilise their left hands in a meaningful way in visuo-constructive tasks such as copying drawings or block-design. Therefore, we conjecture that split-brain patients are capable of using their left hands for the communication of the content of visuo-spatial animations via gestural demonstration. On this basis, we further examine the use of space in communicative gestures by the right and left hands. McNeill and Pedelty (1995) noted for the split-brain patient N.G. that her iconic right hand gestures were exclusively displayed in the right personal space. The present study investigates systematically if there is indication for neglect of the left personal space in right hand gestures in split-brain patients.

\section{MAterials AND Methods}

Our sample comprised three patients who had undergone complete callosotomy because of intractable epilepsy (A.A.: m, age at surgery: 14; age at testing: 50; G.C.: m, 38; 46; N.G.: f, 30; 66; case histories see Bogen, 1969; Milner and Taylor, 1972; Zaidel, 1998). As controls we tested two gender- and age-matched groups, including five patients with partial callosotomy and nine healthy subjects. Each subject was shown 24 animations of two objects moving on a horizontal line that was marked by a centre point and right and left endpoints. For example, an object appears at the centre of the animation scene; the second object appears at the right border of the animation scene, moves towards the centred object, and touches it; then the centred object moves towards the left border of the animation scene. Subjects were asked to demonstrate in gesture and without speaking the content of each animation directly after presentation. The task differed from a mere copying task as the subjects did not represent the events with reference to the screen but they established an imaginative scene in front of themselves. No instruction was given concerning the use of the left and right hands in the gestural demonstrations. The video-taped gesture demonstrations were evaluated by two independent raters who were blind to the subjects' diagnoses and the research hypotheses.

The subjects' spontaneous hand use for the gestural representation of the two objects

Cortex, (2002) 38, 883-886 
was evaluated, separately for the right and left hands: 1 . The hand was not used in the gestural response; 2 . Independent: the hand by itself represented an object, i.e. independently from the other hand; 3. Two-handed: the hand was used only as part of a two-handed object representation, i.e. the two hands jointly represented an object. Per response and hand, the three categories were mutually exclusive. The rater agreement (Cohen's Kappa) for right hand use was $r=.83$ and for left hand use $r=.88$. Separately for the right and left hands, the placement of the borders of the gesturally demonstrated scene was evaluated as follows: 1. Corresponding: the left and right borders of the scene are placed in the corresponding personal spaces, e.g. the left border in the left personal space; 2. Non-corresponding: the left border is shifted to the right, i.e. placed in front of the body-midline or further to the right; 3. Non-corresponding: the right border is shifted to the left, i.e. placed in front of the body-midline or further to the left. The rater agreement (Cohen's Kappa) was $r=.77$.

\section{RESULTS}

\section{Right and Left hand Use in the Gestural Demonstrations}

The subjects' numbers of responses in each hand use category were submitted to a mixed ANOVA with one between-subjects factor (group) and two repeated factors (hand, type of hand use) with two levels each (right hand vs. left hand; independent vs. two-handed). The effects of hand $(\mathrm{F}=5.98 ; \mathrm{df}=1$; $\mathrm{p}=.026)$, type of hand use $(\mathrm{F}=48.86 ; \mathrm{df}=1 ; \mathrm{p}<.001)$, and the interaction of hand $x$ type of hand use $(F=13.63 ; \mathrm{df}=1 ; \mathrm{p}=.002)$ were significant. There were no effects of group and of any interaction with group. Hence, the split-brain patients did not differ in their patterns of hand use from the two control groups.

\section{Placement of the Left and Right Borders of the Gesturally Demonstrated Scene in Relation to the Body-midline and the Right and Left Personal Spaces}

Table I shows the numbers of border placements in the corresponding personal spaces and of border placements with shifts towards the contralateral sides.

With either hand, the two control groups represented the right border of the

TABLE I

Group means and standard deviations of the numbers of border placements in the corresponding personal spaces and of border placements with shifts towards the contralateral sides

\begin{tabular}{|c|c|c|c|c|c|c|}
\hline & \multicolumn{3}{|c|}{ Left hand } & \multicolumn{3}{|c|}{ Right hand } \\
\hline & \multirow{2}{*}{$\begin{array}{c}\text { corresponding } \\
\text { border } \\
\text { placement }\end{array}$} & \multicolumn{2}{|c|}{ shifts } & \multirow{2}{*}{$\begin{array}{c}\text { corresponding } \\
\text { border } \\
\text { placement }\end{array}$} & \multicolumn{2}{|c|}{ shifts } \\
\hline & & $\begin{array}{l}\text { left border } \\
\text { to the right }\end{array}$ & $\begin{array}{l}\text { right border } \\
\text { to the left }\end{array}$ & & $\begin{array}{l}\text { left border } \\
\text { to the right }\end{array}$ & $\begin{array}{l}\text { right border } \\
\text { to the left }\end{array}$ \\
\hline $\begin{array}{l}\text { Complete } \\
\text { Callosotomy }\end{array}$ & $13.7 \pm 8.5$ & $.0 \pm .0$ & $.0 \pm .0$ & $24.7 \pm 2.6$ & $5.7 \pm 4.0^{*}$ & $0.4 \pm 0.6$ \\
\hline $\begin{array}{l}\text { Partial } \\
\text { Callosotomy }\end{array}$ & $11.4 \pm 10.6$ & $.0 \pm .0$ & $.0 \pm .0$ & $23.5 \pm 12.9$ & $0.6 \pm 1.3$ & $.0 \pm .0$ \\
\hline $\begin{array}{l}\text { Healthy } \\
\text { Subjects }\end{array}$ & $15.7 \pm 7.4$ & $.0 \pm .0$ & $0.1 \pm 0.3$ & $24.4 \pm 8.1$ & $0.1 \pm 0.3$ & $.0 \pm .0$ \\
\hline
\end{tabular}

Note: corresponding border placement $=$ hand places the left and right border in the corresponding left and right personal spaces; $*=$ significant interaction. 
scene in their right personal spaces and the left border in their left personal spaces. In their right hand demonstrations, the split-brain patients shifted the left borders of the scene to the right. In fact, they placed the left borders in front of their body-midlines and never further to the contralateral right sides. With their left hands, the split-brain patients showed the same performances as the two control groups.

For each hand, the proportions of shifts of the border ipsilateral to the performing hand and of shifts of the border contralateral to the performing hand were calculated. For example, for the right hand, the number of ipsilateral right border shifts were divided by the total number of right hand border placements. The subjects' proportions were submitted to a mixed ANOVA with one betweensubjects factor (group) and two within-subjects factors (hand, shifted border) with two levels each (right vs. left hand; border ipsilateral vs. border contralateral to performing hand). There were significant effects of group $(\mathrm{F}=22.65 ; \mathrm{df}=2$; $\mathrm{p}<.001)$, hand $(\mathrm{F}=46.28 ; \mathrm{df}=1 ; \mathrm{p}<.001)$; hand $\times$ group $(\mathrm{F}=40.0 ; \mathrm{df}=2$; $\mathrm{p}<.001)$, shifted border $(\mathrm{F}=33.25 ; \mathrm{df}=1 ; \mathrm{p}<.001)$, shifted border $\mathrm{x}$ group $(\mathrm{F}=21.72 ; \mathrm{df}=2 ; \mathrm{p}<.001)$, hand $\times$ shifted border $(\mathrm{F}=28.95 ; \mathrm{df}=1 ; \mathrm{p}<.001)$, and hand $\times$ shifted border $\times$ group $(\mathrm{F}=16.01, \mathrm{df}=2, \mathrm{p}<.001)$.

\section{Discussion}

The split-brain patients did not differ from the two control groups in their use of the right and left hands in the gestural demonstrations of the animations. Their left hand ability to express spatial information in communicative gestures contrasted their left hand dyspraxia in tasks that require language processing and in pantomime of object use to visual presentation of objects (Lausberg et al., in press). We assume that in the present experiment, the visuo-spatial quality of the animation enabled the right hemisphere to act motorically in a meaningful manner. A comparable creative use of the left hand had been observed in the block design experiment. Hence, our findings concur with the proposition that in split-brain patients each hemisphere has the potential to exert volitional motor control if the task requests functions for which the respective hemisphere is specialized (Gazzaniga et al., 1967; Zaidel and Sperry, 1977).

In the split-brain patients, hemispheric specialization in the use of space was evidenced by differences between the right hand and left hand gestural demonstrations. In their right hand demonstrations, the split-brain patients differed significantly from the patients with partial callosotomy and the healthy controls as they shifted the left border of the whole scene to the right and placed it in front of their body-midlines. With their left hands, they showed the same performances as the two control groups with either hand, i.e. they placed the left and right borders of the scene in the corresponding personal spaces. This finding implies a dissociation of the gesture spaces of the two hands.

Our findings consolidate McNeill and Pedelty's observation (1995) and indicate a neglect of the left personal space for gestural demonstrations by the right hand in split-brain patients. Thus far, McNeill and Pedelty's finding stood apart from the general opinion that no salient left neglect by the separate left 
hemisphere existed in patients with complete callosotomy, and that the disconnected left hemisphere by itself possessed adequate mechanisms for attending to and acting in both halves of the space (Plourde and Sperry, 1984). In contrast, the present findings on the spontaneous use of space in communicative gestures in split-brain patients suggest similar patterns of hemispheric specialisation concerning the use of space as described for patients with spontaneous callosal disconnection (Sine et al., 1984; Goldenberg, 1986; Kashiwagi et al., 1990).

To summarise, our data provide evidence that the two separate hemispheres create different gesture spaces, with the right hemisphere using the right and left personal spaces and the left hemisphere showing a preponderance for the right personal space.

Acknowledgements. This study was supported by the German Research Association grants LA 1249/1-1 and LA 1249/1-2, and by the Language and Cognition Group of the Max Planck Institute for Psycholinguistics. In particular, we wish to thank the raters for the coding of the extensive video material, and the patients and the controls for their participation in the study.

\section{REFERENCES}

BogEn JE. The other side of the brain I: Dysgraphia and dyscopia following cerebral commissurotomy. Bulletin of the Los Angeles Neurological Societies, 34: 73-105, 1969.

GAZZANIGA MS, BOGEn JE and SPERRY RW. Dyspraxia following division of the cerebral commissures. Archives of Neurology, 16: 606-612, 1967.

GOLDENBERG G. Neglect in a patient with partial callosal disconnection. Neuropsychologia, 24: 397-403, 1986.

Kashiwagi A, Kashiwagi T, Nishikawa T, Tanabe H and OKuda J. Hemispatial neglect in a patient with callosal infarction. Brain, 113: 1005-1023, 1990.

Lausberg H, Cruz RF, Kita S, Zaidel E and Ptito A. Pantomime to visual presentation of objects: left and dyspaxia in patients with complete callostomy. Brain, in press.

McNeill D and Pedelty LL. Right brain and gesture. In K Emmorey and J Reilty (Eds), Language, Gesture and Space. Hove, Hillsdale: Erlbaum, 1995, pp. 63-85.

MILNER B and TAYLOR L. Right-hemisphere superiority in tactile pattern-recognition after cerebral commissurotomy: Evidence for non-verbal memory. Neuropsychologia, 10: 1-15, 1972.

Plourde G and SPERRY RW. Left hemisphere involvement in left spatial neglect from right-sided lesions. Brain, 107: 95-106, 1984.

Sine RD, SoufI A and SHAH M. Callosal syndrome: Implications for understanding the neuropsychology of stroke. Archives of Physical Medicine and Rehabilitation, 65: 606-610, 1984.

ZAIDEL E. Stereognosis in the chronic split brain: Hemispheric differences, ipsilateral control and sensory integration across the midline. Neuropsychologia, 36: 1033-1047, 1998.

ZAIDEL E and SPERRY RW. Some long-term motor effects of cerebral commissurotomy in man. Neuropsychologia, 15: 193-204, 1977. 